\title{
Serum Anion Gap Predicts All-Cause Mortality in Critically Ill Patients with Acute Kidney Injury: Analysis of the MIMIC- III Database
}

\author{
Bihuan Cheng $\mathbb{D}^{D}$, Diwen Li, Yuqiang Gong $\mathbb{D}^{D}$, Binyu Ying, and Benji Wang $\mathbb{D}$ \\ Department of Anesthesiology, Critical Care and Pain Medicine, The Second Affiliated Hospital and Yuying Children's Hospital of \\ Wenzhou Medical University, Wenzhou, 325000 Zhejiang, China \\ Correspondence should be addressed to Benji Wang; wbj@wmu.edu.cn
}

Received 8 August 2019; Revised 16 December 2019; Accepted 9 January 2020; Published 21 January 2020

Academic Editor: Alexandra Scholze

Copyright () 2020 Bihuan Cheng et al. This is an open access article distributed under the Creative Commons Attribution License, which permits unrestricted use, distribution, and reproduction in any medium, provided the original work is properly cited.

\begin{abstract}
Background. No epidemiological study has investigated the effect of anion gap (AG) on the prognosis of critically ill patients with acute kidney injury (AKI). Therefore, we aimed to determine the association between serum AG and all-cause mortality in these patients. Methods. From MIMIC III, we extracted demographics, vital signs, laboratory tests, comorbidities, and scoring systems from the first $24 \mathrm{~h}$ after patient ICU admission. A generalized additive model was used to identify a nonlinear association between anion gap and 30-day all-cause mortality. We also used the Cox proportional hazards models to measure the association between AG levels and 30-day, 90-day, and 365-day mortality in patients with AKI. Results. A total of 11,573 eligible subjects were extracted from the MIMIC-III. The relationship between AG levels and 30day all-cause mortality in patients with AKI was nonlinear, with a U-shaped curve. In multivariate analysis, after adjusting for potential confounders, higher AG was a significant predictor of 30-day, 90-day, and 365-day all-cause mortality compared with lower AG (HR, 95\% CI: 1.54, 1.33-1.75; 1.55, 1.38-1.73; 1.46, 1.31-1.60). Conclusions. The relationship between AG levels and 30-day all-cause mortality described a U-shaped curve. High-AG levels were associated with increased risk 30-day, 90-day, and 365-day all-cause mortality in critically ill patients with AKI.
\end{abstract}

\section{Introduction}

Acute kidney injury (AKI) is a common syndrome characterized by an abrupt, usually reversible decline in glomerular filtration, associated with substantial morbidity and mortality, especially in critically ill patients $[1,2]$. In the US, approximately $20 \%$ of critically ill patients have AKI in the intensive care unit (ICU) [3]. In the presence of AKI, patient mortality within 1 year after ICU admission is significantly elevated, as high as $60 \%-$ $70 \%[4,5]$. Because of the high incidence and poor prognosis of AKI in critically ill patients, researchers are attempting to identify prognostic predictors in AKI $[6,7]$. Unfortunately, most of them are not widely used in clinical practice.
The serum anion gap $(\mathrm{AG})$ is a mathematical derivation parameter calculated from the difference in serum cation and anion concentrations. It is the simplest way to assess acid-base status $[8,9]$ and helps to identify various forms of metabolic acidosis. AG is also one of the most commonly used biomarkers that provides important clues regarding diagnosis or prognosis of various disorders [10-12]. There is a positive monotonic relationship between high AG and severity of illness or poor prognosis for sepsis [13], coronary artery disease (CAD) [14], aortic aneurysm [15], and chronic kidney disease (CKD) [16]. To the best of our knowledge, no epidemiological study has investigated the effect of AG on the prognosis of critically ill patients with AKI. Therefore, we aimed to determine the association between serum AG and all-cause mortality in these patients. 


\section{Methods}

2.1. Data Source. We followed the methods of Wang et al. in this study, as we have done previously [17-19]. Our study is based on an openly available clinical database called the Multiparameter Intelligent Monitoring in Intensive Care III (MIMIC III) [20]. The database includes more than 40,000 ICU patients admitted to Beth Israel Deaconess Medical Center (Boston, MA, USA) from 2001 to 2012. To apply for access to the database, we completed the National Institutes of Health's web-based course and passed the Protecting Human Research Participants exam (no. 6182750). We extracted clinical variables, including demographic characteristics, International Classification of Diseases (ICD-9) codes, physiological index, medications, and laboratory tests. The project was approved by the Institutional Review Boards of Beth Israel Deaconess Medical Center and the Massachusetts Institute of Technology (Cambridge, MA). To safeguard patient privacy, data were deidentified; therefore, informed consent was waived.

2.2. Population Selection Criteria. Adult patients ( $\geq 18$ years) with AKI according to ICD-9 code at first ICU admission for more than two days were included. The exclusion criteria were (1) missing AG at ICU admission and (2) missing $>5 \%$ individual data.

2.3. Data Extraction. Structured Query Language (SQL) with PostgreSQL (version 9.6) was used to extract data from MIMIC-III. Demographics, vital signs, laboratory tests, comorbidities, scoring systems, and other variables collected within the first $24 \mathrm{~h}$ of ICU admission were extracted from MIMIC III. The comorbidities included CAD, congestive heart failure (CHF), atrial fibrillation (AFIB), stroke, renal disease, liver disease, pneumonia, respiratory failure, and acute respiratory distress syndrome (ARDS). Laboratory data were also extracted, including AG, albumin, bicarbonate, bilirubin, creatinine, chloride, glucose, hematocrit, hemoglobin, platelet, sodium, potassium, lactate, blood urea nitrogen (BUN), white blood cell (WBC), prothrombin time (PT), activated partial thromboplastin time (APTT), and international normalized ratio (INR). We also calculated the sequential organ failure assessment (SOFA) score [21] and simplified acute physiology score II (SAPSII) [22] for each patient. Other extracted data included age, gender, systolic blood pressure (SBP), diastolic blood pressure (DBP), mean blood pressure (MBP), heart rate, respiratory rate, temperature, SPO2, AKI stage, renal replacement therapy, and ICU length of stay (LOS). Survival information on vital status was obtained from Social Security Death Index records. The endpoints of our study were 30-day, 90-day, and 365-day all-cause mortality from the date of ICU admission.

2.4. Statistical Analysis. Continuous variables were expressed as mean $\pm \mathrm{SD}$ or medians and interquartile range (IQR). Categorical data were expressed as frequencies or percentages. Chi-square, 1-way ANOVA, and Kruskal-Wallis $H$ tests were used to determine any significant differences between the groups. A generalized additive model (GAM) was used to identify the nonlinear association between AG and 30-day all-cause mortality. We also used the Cox proportional hazards models to determine associations between AG levels and 30-day, 90-day, and 365-day mortality in AKI; these results were expressed as hazard ratios (HRs) with $95 \%$ confidence intervals (CIs).

Variables based on epidemiological and biological background were incorporated as potential confounders, and those confounders based on a change in effect estimate of $>10 \%$ were used to generate an adjusted model [23]. For each endpoint, two multivariate models were constructed on the basis of AG group inclusion according to tertiles. The first tertile was treated as the reference group. In model I, covariates were adjusted for age and gender. In model II, we further adjusted for age, gender, AKI stage, $\mathrm{CHF}$, CAD, liver disease, stroke, respiratory failure, pneumonia, SIRS, potassium, albumin, lactate, platelet, BUN, PT, INR, APTT, WBC, $\mathrm{pH}$, creatinine, bicarbonate, bilirubin, renal replacement therapy, respiration rate, SPO2, heart rate, SBP, DBP, temperature, Elixhauser comorbidity index, SOFA, and SAPSII. Subgroup analysis of the associations between AG and 30-day all-cause mortality was performed using stratified linear regression models. All probability values were 2 -sided, and values less than 0.05 were considered statistically significant. R (http://www.R-project.org) and EmpowerStats (http://www.empowerstats.com/en/, X\&Y solutions, Inc., Boston, MA) were used for all statistical analysis.

\section{Results}

3.1. Subject Characteristics. The patients were divided according to AG in tertiles, and baseline characteristics of these patients are summarized in Table 1. A total of 11,573 eligible subjects were extracted from the MIMIC-III. There were 6,626 men and 4,947 women, and patients were generally older. Patients with higher $A G(A G \geq 14)$ were more likely to report a history of CAD, CHF, AFIB, and renal disease and had higher values of bilirubin, creatinine, potassium, lactate, BUN, WBC, PT, and APTT. SAPSII, SOFA scores, mortality, use of RRT, and ICU LOS were also significantly higher in the high-AG group $(A G \geq 14)$ than in the lower-AG group $(\mathrm{AG}<12)$.

3.2. AG Levels and All-Cause Mortality. The relationship between AG levels and 30-day all-cause mortality was nonlinear, and a U-shaped curve was observed (Figure 1). We used Cox proportional hazards regression model to determine the associations between AG and 30-day, 90-day, and 365-day all-cause mortality in patients with AKI (Table 2). In model $\mathrm{I}$, high-AG levels $(A G \geq 14)$ were associated with increased risk of all-cause mortality after adjustment for age and gender. In model II, the lower AG $(A G<12)$ was used as a reference. After adjustment for confounders (age, gender, acute kidney injury stage, congestive heart failure, coronary artery disease, liver disease, stroke, respiratory failure, pneumonia, SIRS, potassium, albumin, lactate, platelet, BUN, PT, INR, APTT, WBC, pH, creatinine, bicarbonate, sodium, chloride, diabetes, bilirubin, renal replacement therapy, respiration rate, SPO2, heart rate, SBP, DBP, temperature, Elixhauser comorbidity index, SOFA, and SAPSII), 
TABLE 1: Baseline characteristics of participants according to anion gap $(N=11,573)$.

\begin{tabular}{|c|c|c|c|c|}
\hline \multirow{2}{*}{ Characteristic } & \multicolumn{3}{|c|}{ Anion gap (mmol/L) } & \multirow{2}{*}{$P$ value } \\
\hline & Q1 $(<12)$ & Q2 $(\geq 12-<14)$ & Q3 $(\geq 14)$ & \\
\hline Age (years) & $62.4 \pm 17.3$ & $64.5 \pm 16.9$ & $64.9 \pm 16.6$ & $<0.001$ \\
\hline Gender, $n(\%)$ & & & & 0.653 \\
\hline Female & $1432(42.2)$ & $1312(43.3)$ & $2203(42.8)$ & \\
\hline Male & $1964(57.8)$ & $1718(56.7)$ & $2944(57.2)$ & \\
\hline MBP (mmHg) & $77.5 \pm 11.2$ & $77.8 \pm 11.5$ & $77.7 \pm 12.8$ & 0.316 \\
\hline Heart rate (beats/minute) & $87.9 \pm 16.9$ & $87.5 \pm 16.8$ & $89.0 \pm 17.3$ & $<0.001$ \\
\hline Respiratory rate (beats/minute) & $18.9 \pm 4.1$ & $19.4 \pm 4.2$ & $20.1 \pm 4.4$ & $<0.001$ \\
\hline Temperature $\left({ }^{\circ} \mathrm{C}\right)$ & $36.9 \pm 0.7$ & $36.9 \pm 0.7$ & $36.8 \pm 0.7$ & $<0.001$ \\
\hline SPO2 (\%) & $97.4 \pm 2.0$ & $97.3 \pm 2.2$ & $97.0 \pm 2.8$ & $<0.001$ \\
\hline \multicolumn{5}{|l|}{ Comorbidities, $n(\%)$} \\
\hline Coronary artery disease & $719(21.2)$ & $709(23.4)$ & $1224(23.8)$ & 0.015 \\
\hline Congestive heart failure & $503(14.8)$ & $540(17.8)$ & $1131(22.0)$ & $<0.001$ \\
\hline Atrial fibrillation & $774(22.8)$ & $858(28.3)$ & $1546(30.0)$ & $<0.001$ \\
\hline Stroke & $224(6.6)$ & $275(9.1)$ & $408(7.9)$ & 0.001 \\
\hline Renal disease & $387(11.4)$ & $479(15.8)$ & $1381(26.8)$ & $<0.001$ \\
\hline Liver disease & $470(13.8)$ & $315(10.4)$ & $605(11.8)$ & $<0.001$ \\
\hline Respiratory failure & $1406(41.4)$ & $1212(40.0)$ & $2141(41.6)$ & 0.339 \\
\hline ARDS & $62(1.8)$ & $75(2.5)$ & $135(2.6)$ & 0.051 \\
\hline \multicolumn{5}{|l|}{ Laboratory parameters } \\
\hline Anion gap $(\mathrm{mmol} / \mathrm{L})$ & $9.8 \pm 1.4$ & $12.5 \pm 0.5$ & $16.5 \pm 2.9$ & $<0.001$ \\
\hline Albumin $(\mathrm{g} / \mathrm{dL})$ & $3.1 \pm 0.7$ & $3.2 \pm 0.7$ & $3.2 \pm 0.7$ & $<0.001$ \\
\hline Bicarbonate (mmol/L) & $26.8 \pm 5.1$ & $24.8 \pm 4.1$ & $22.6 \pm 4.5$ & $<0.001$ \\
\hline Bilirubin (mg/dL) & $1.8 \pm 3.5$ & $1.7 \pm 3.9$ & $2.6 \pm 6.0$ & $<0.001$ \\
\hline Creatinine $(\mathrm{mEq} / \mathrm{L})$ & $1.3 \pm 0.8$ & $1.7 \pm 1.2$ & $3.0 \pm 2.8$ & $<0.001$ \\
\hline Chloride (mmol/L) & $109.2 \pm 7.5$ & $108.2 \pm 6.9$ & $105.9 \pm 6.9$ & $<0.001$ \\
\hline Glucose (mg/dL) & $202.1 \pm 150.4$ & $201.4 \pm 134.7$ & $203.7 \pm 130.3$ & 0.005 \\
\hline Hematocrit (\%) & $35.5 \pm 6.2$ & $35.7 \pm 6.0$ & $35.2 \pm 6.1$ & $<0.001$ \\
\hline Hemoglobin $(\mathrm{g} / \mathrm{dL})$ & $11.8 \pm 2.1$ & $11.9 \pm 2.1$ & $11.7 \pm 2.1$ & $<0.001$ \\
\hline Platelet $\left(10^{9} / \mathrm{L}\right)$ & $236.2 \pm 129.5$ & $253.8 \pm 143.3$ & $251.6 \pm 137.7$ & $<0.001$ \\
\hline Sodium $(\mathrm{mmol} / \mathrm{L})$ & $141.0 \pm 6.0$ & $140.7 \pm 5.3$ & $140.1 \pm 5.4$ & $<0.001$ \\
\hline Potassium (mmol/L) & $4.7 \pm 0.9$ & $4.7 \pm 1.0$ & $5.0 \pm 1.1$ & $<0.001$ \\
\hline Lactate $(\mathrm{mmol} / \mathrm{L})$ & $3.0 \pm 2.5$ & $3.3 \pm 2.7$ & $3.9 \pm 3.4$ & $<0.001$ \\
\hline $\mathrm{BUN}(\mathrm{mg} / \mathrm{dL})$ & $27.6 \pm 17.6$ & $32.5 \pm 20.8$ & $49.1 \pm 33.3$ & $<0.001$ \\
\hline WBC $\left(10^{9} / \mathrm{L}\right)$ & $14.0 \pm 12.8$ & $14.8 \pm 13.4$ & $16.0 \pm 12.9$ & $<0.001$ \\
\hline PT (seconds) & $17.6 \pm 9.1$ & $17.9 \pm 11.4$ & $19.8 \pm 14.0$ & $<0.001$ \\
\hline APTT (seconds) & $46.9 \pm 32.3$ & $46.2 \pm 32.1$ & $49.8 \pm 34.3$ & $<0.001$ \\
\hline \multicolumn{5}{|l|}{ Scoring systems } \\
\hline SOFA & $5.1 \pm 3.1$ & $5.1 \pm 3.3$ & $6.4 \pm 3.8$ & $<0.001$ \\
\hline SAPSII & $37.7 \pm 13.6$ & $38.9 \pm 14.0$ & $44.0 \pm 15.3$ & $<0.001$ \\
\hline AKI stage, $n(\%)$ & & & & $<0.001$ \\
\hline Stage 1 & $838(24.7)$ & $727(24.0)$ & $1011(19.6)$ & \\
\hline Stage 2 & $705(20.8)$ & $550(18.2)$ & $689(13.4)$ & \\
\hline Stage 3 & $1853(54.6)$ & $1753(57.9)$ & $3447(67.0)$ & \\
\hline Renal replacement therapy, $n(\%)$ & $154(4.5)$ & $209(6.9)$ & $1120(21.8)$ & $<0.001$ \\
\hline ICU LOS (days) & $5.9 \pm 7.0$ & $6.1 \pm 7.2$ & $6.6 \pm 8.4$ & $<0.001$ \\
\hline
\end{tabular}


TABLE 1: Continued.

\begin{tabular}{|c|c|c|c|c|}
\hline \multirow{2}{*}{ Characteristic } & \multicolumn{3}{|c|}{ Anion gap (mmol/L) } & \multirow{2}{*}{$P$ value } \\
\hline & Q1 $(<12)$ & $\mathrm{Q} 2(\geq 12-<14)$ & Q3 $(\geq 14)$ & \\
\hline 30-day mortality, $n(\%)$ & $516(15.2)$ & $512(16.9)$ & $1361(26.4)$ & $<0.001$ \\
\hline 90-day mortality, $n(\%)$ & $737(21.7)$ & $729(24.1)$ & $1796(34.9)$ & $<0.001$ \\
\hline 365-day mortality, $n$ (\%) & $1058(31.2)$ & $1057(34.9)$ & $2305(44.8)$ & $<0.001$ \\
\hline
\end{tabular}

MBP: mean blood pressure; ARDS: acute respiratory distress syndrome; BUN: blood urea nitrogen; WBC: white blood cell; PT: prothrombin time; APTT: activated partial thromboplastin time; SOFA: sequential organ failure assessment; SAPSII: simplified acute physiology score II; AKI: acute kidney injury; ICU: intensive care unit; LOS: length of stay.

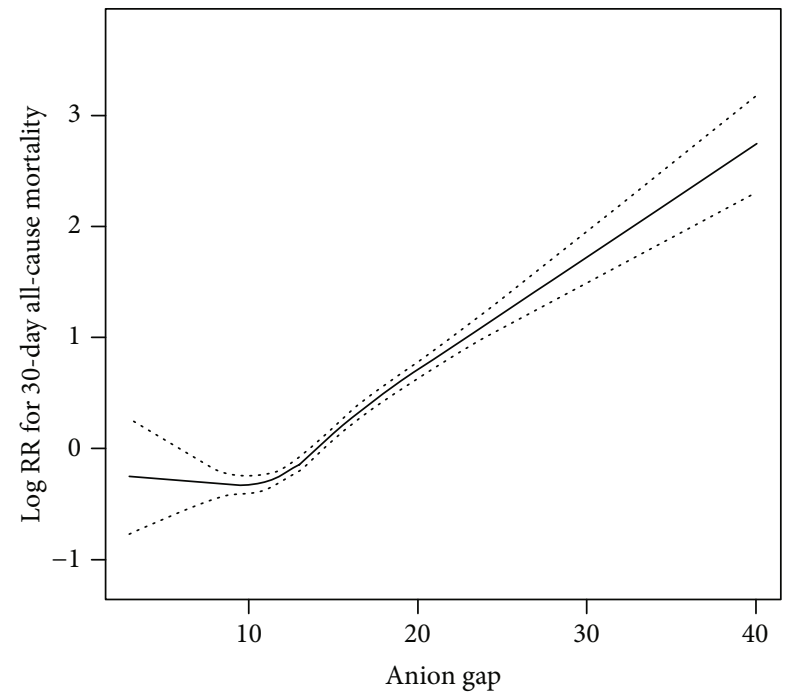

Figure 1: Association between anion gap and 30-day all-cause mortality. A threshold, nonlinear association between anion gap and 30-day all-cause mortality was found in a generalized additive model (GAM). Solid rad line represents the smooth curve fit between variables. Imaginary lines represent the $95 \%$ of confidence interval from the fit.

higher $A G(A G \geq 14)$ remained a more significant predictor of 30-day, 90-day, and 365-day all-cause mortality than lower AG (HR, 95\% CI: 1.54, 1.33-1.75; 1.55, 1.38-1.73; 1.46, 1.31$1.60)$. For the purpose of sensitivity analysis, we also handled AG as categorical variable (tertile) and found the same trend ( $P$ for trend: $<0.0001$ ). In addition, in order to verify that AG was an independent prognostic factor for AKI, we also analyzed the potential associations of bicarbonate, $\mathrm{pH}$, lactate, and urine ketone bodies on all-cause mortality, and the results were included in supplementary material (available here).

3.3. Subgroup Analyses. As shown in Table 3, the test for interactions was statistically significant in several strata ( $P$ for interaction $<0.05$ ). Among these strata, we observed that patients with higher AGs had significantly higher mortality with hypotension, bicarbonate $<24 \mathrm{mg} / \mathrm{dL}$, bilirubin $\geq 0.7 \mathrm{mg} / \mathrm{dL}, \quad$ lactate $\geq 2.5 \mathrm{mmol} / \mathrm{L}, \quad \mathrm{PT} \geq 15.2 \mathrm{~s}$, INR $\geq 1.4$, APTT $\geq 34.8 \mathrm{~s}, \mathrm{WBC} \geq 13 \times 10^{9} / \mathrm{L}$, creatinine $\geq$ $1.4 \mathrm{mEq} / \mathrm{L}$, and chloride $\geq 107 \mathrm{mmol} / \mathrm{L}$. Similar trends were observed in patients with $\mathrm{CAD}$ and liver disease.

\section{Discussion}

The relationship between AG and 30-day all-cause mortality among critically ill patients with AKI was nonlinear, and a U-shaped curve was observed. In the fully adjusted model, high-AG levels were associated with increased risk 30-day, 90-day, and 365-day all-cause mortality. To our knowledge, this was the first study to measure the association between serum AG and all-cause mortality in critically ill patients with AKI.

Several studies have explored the relationship between AG and clinical outcomes of various diseases. Yang et al. [14] measured the association between the AG and allcause mortality in CAD and found that higher AG was associated with worse cardiac function and was a significant predictor of all-cause mortality. Banerjee et al. [24] suggested that greater AG was present among persons with $\mathrm{CKD}$, and $\mathrm{AG}$ increased the risk for progression to end-stage renal disease in these patients. AG is a traditional tool for assessing acid-base status, and most previous studies have associated it with acid-base disorders, all of which have significant impacts on morbidity and mortality in critically ill patients [25]. Similarly, our findings showed a positive correlation between serum AG and allcause mortality in critically ill patients with AKI. Nevertheless, the underlying mechanism requires further research.

AG reflects the unmeasured anion concentration and can be easily calculated from conventional clinical chemical analysis. It is widely used to evaluate the acid-base status and is one of the most commonly used biomarkers that provides important clues for the diagnosis and prognosis of various diseases $[13,15]$. Elevated serum AG is usually caused by overproduction of organic acid anions and/or reduction in anion excretion [8]; increased serum lactate and ketoanions are the main reasons for increased AG [26]. AKI is defined as rapid decline in renal function lasting from hours to days, as opposed to chronic kidney disease [27]. Critically ill patients are often exposed to hypoxia and anaerobic tissue conditions, leading to rapid accumulation of pyruvate, which is almost completely converted to lactate [28]. The kidney is known for lactate dehydrogenase dysfunction in the setting of patients with AKI [29], and the renal acid excretion does not fully offset endogenous acid production [30]. Consequently, high AG is common in critically ill patients with AKI. 


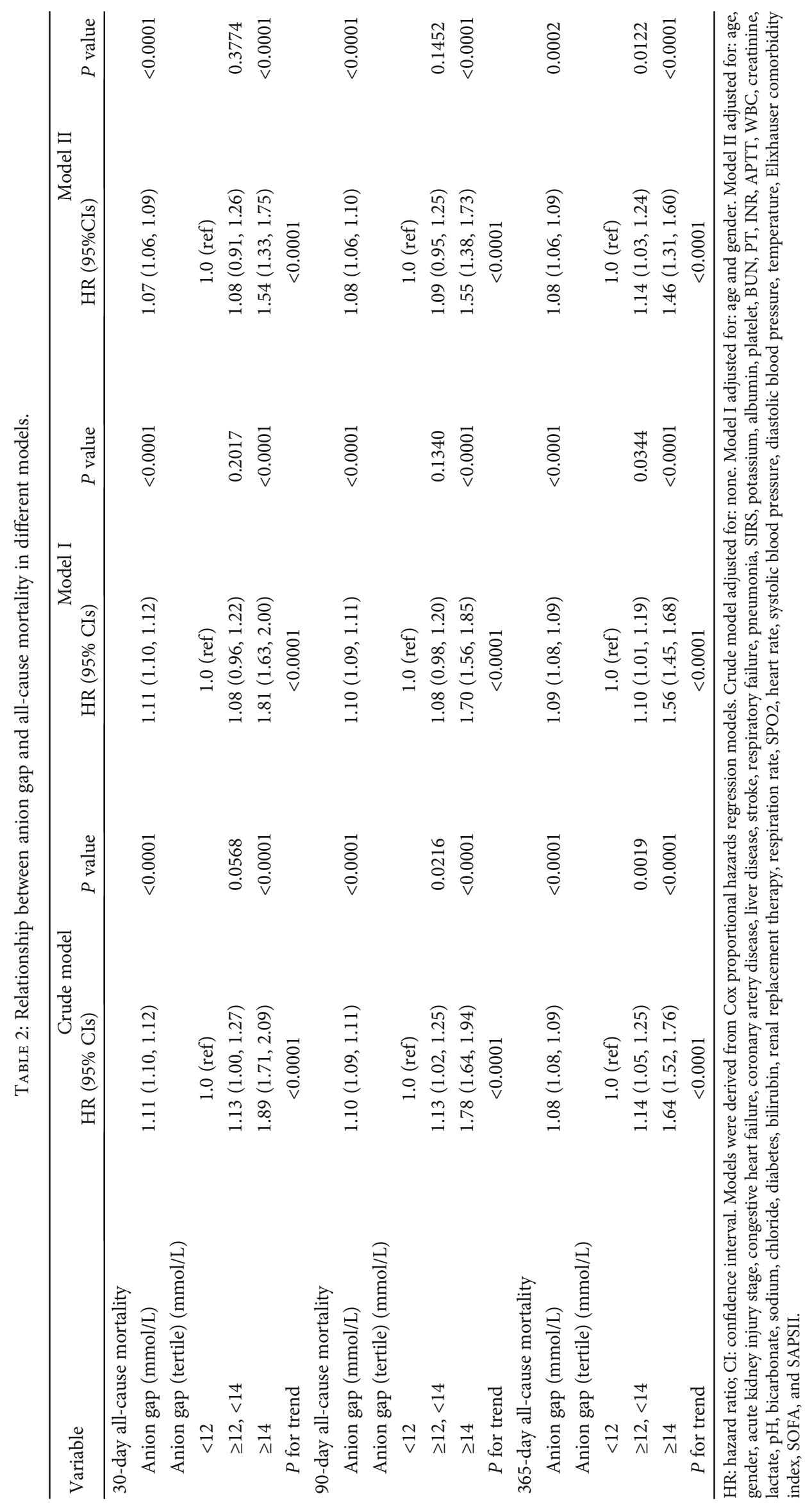


TABLE 3: Subgroup analysis of the associations between anion gap and 30-day all-cause mortality.

\begin{tabular}{|c|c|c|c|c|}
\hline Characteristic & $N$ & HR (95\% CI) & $P$ value & $P$ for interaction \\
\hline $\mathrm{CHF}$ & & & & 0.0848 \\
\hline No & 9399 & $1.12(1.11,1.13)$ & $<0.0001$ & \\
\hline Yes & 2174 & $1.09(1.06,1.12)$ & $<0.0001$ & \\
\hline CAD & & & & 0.0056 \\
\hline No & 8921 & $1.10(1.09,1.12)$ & $<0.0001$ & \\
\hline Yes & 2652 & $1.15(1.12,1.18)$ & $<0.0001$ & \\
\hline AFIB & & & & 0.0023 \\
\hline No & 8395 & $1.12(1.11,1.14)$ & $<0.0001$ & \\
\hline Yes & 3178 & $1.09(1.07,1.11)$ & $<0.0001$ & \\
\hline Renal disease & & & & 0.4628 \\
\hline No & 9326 & $1.12(1.11,1.13)$ & $<0.0001$ & \\
\hline Yes & 2247 & $1.11(1.09,1.14)$ & $<0.0001$ & \\
\hline Liver disease & & & & 0.0436 \\
\hline No & 10183 & $1.11(1.09,1.12)$ & $<0.0001$ & \\
\hline Yes & 1390 & $1.14(1.11,1.16)$ & $<0.0001$ & \\
\hline Stroke & & & & 0.0184 \\
\hline No & 10666 & $1.12(1.11,1.13)$ & $<0.0001$ & \\
\hline Yes & 907 & $1.07(1.03,1.11)$ & 0.0002 & \\
\hline Pneumonia & & & & $<0.0001$ \\
\hline No & 7997 & $1.14(1.12,1.15)$ & $<0.0001$ & \\
\hline Yes & 3576 & $1.06(1.05,1.08)$ & $<0.0001$ & \\
\hline Respiratory failure & & & & 0.0002 \\
\hline No & 6814 & $1.13(1.12,1.15)$ & $<0.0001$ & \\
\hline Yes & 4759 & $1.09(1.08,1.11)$ & $<0.0001$ & \\
\hline ARDS & & & & 0.8864 \\
\hline No & 11301 & $1.11(1.10,1.12)$ & $<0.0001$ & \\
\hline Yes & 272 & $1.13(1.06,1.19)$ & $<0.0001$ & \\
\hline AKI stage & & & & 0.0005 \\
\hline Stage 1 & 2576 & $1.13(1.10,1.16)$ & $<0.0001$ & \\
\hline Stage 2 & 1944 & $1.05(1.02,1.09)$ & 0.0032 & \\
\hline Stage 3 & 7053 & $1.11(1.10,1.13)$ & $<0.0001$ & \\
\hline Albumin $(\mathrm{g} / \mathrm{dL})$ & & & & 0.3737 \\
\hline$<3.2$ & 5614 & $1.11(1.10,1.13)$ & $<0.0001$ & \\
\hline$\geq 3.2$ & 5959 & $1.12(1.10,1.14)$ & $<0.0001$ & \\
\hline Bicarbonate $(\mathrm{mmol} / \mathrm{L})$ & & & & 0.0004 \\
\hline$<24$ & 5009 & $1.12(1.10,1.13)$ & $<0.0001$ & \\
\hline$\geq 24$ & 6564 & $1.07(1.05,1.09)$ & $<0.0001$ & \\
\hline Bilirubin (mg/dL) & & & & $<0.0001$ \\
\hline$<0.7$ & 4674 & $1.07(1.05,1.09)$ & $<0.0001$ & \\
\hline$\geq 0.7$ & 5521 & $1.13(1.12,1.14)$ & $<0.0001$ & \\
\hline Sodium (mmol/L) & & & & 0.0608 \\
\hline$<140$ & 4737 & $1.12(1.10,1.13)$ & $<0.0001$ & \\
\hline$\geq 140$ & 6836 & $1.11(1.09,1.12)$ & $<0.0001$ & \\
\hline Potassium (mmol/L) & & & & 0.4309 \\
\hline$<4.6$ & 5580 & $1.10(1.09,1.12)$ & $<0.0001$ & \\
\hline$\geq 4.6$ & 5993 & $1.11(1.10,1.12)$ & $<0.0001$ & \\
\hline
\end{tabular}


TABle 3: Continued.

\begin{tabular}{|c|c|c|c|c|}
\hline Characteristic & $N$ & $\operatorname{HR}(95 \% \mathrm{CI})$ & $P$ value & $P$ for interaction \\
\hline Lactate $(\mathrm{mmol} / \mathrm{L})$ & & & & $<0.0001$ \\
\hline$<2.5$ & 4334 & $1.06(1.03,1.08)$ & $<0.0001$ & \\
\hline$\geq 2.5$ & 4416 & $1.12(1.11,1.14)$ & $<0.0001$ & \\
\hline BUN (mg/dL) & & & & 0.0610 \\
\hline$<30$ & 5689 & $1.12(1.10,1.15)$ & $<0.0001$ & \\
\hline$\geq 30$ & 5884 & $1.09(1.08,1.10)$ & $<0.0001$ & \\
\hline PT (seconds) & & & & $<0.0001$ \\
\hline$<15.2$ & 5464 & $1.07(1.05,1.09)$ & $<0.0001$ & \\
\hline$\geq 15.2$ & 5602 & $1.12(1.11,1.13)$ & $<0.0001$ & \\
\hline INR & & & & $<0.0001$ \\
\hline$<1.4$ & 4976 & $1.07(1.04,1.09)$ & $<0.0001$ & \\
\hline$\geq 1.4$ & 6088 & $1.11(1.10,1.13)$ & $<0.0001$ & \\
\hline APTT (seconds) & & & & $<0.0001$ \\
\hline$<34.8$ & 5501 & $1.07(1.05,1.09)$ & $<0.0001$ & \\
\hline$\geq 34.8$ & 5544 & $1.12(1.11,1.13)$ & $<0.0001$ & \\
\hline $\mathrm{WBC}\left(10^{9} / \mathrm{L}\right)$ & & & & 0.0245 \\
\hline$<13$ & 5755 & $1.10(1.09,1.12)$ & $<0.0001$ & \\
\hline$\geq 13$ & 5808 & $1.11(1.10,1.12)$ & $<0.0001$ & \\
\hline Platelet $\left(10^{9} / \mathrm{L}\right)$ & & & & 0.0888 \\
\hline$<226$ & 5758 & $1.12(1.11,1.13)$ & $<0.0001$ & \\
\hline$\geq 226$ & 5805 & $1.10(1.08,1.12)$ & $<0.0001$ & \\
\hline Hematocrit (\%) & & & & $<0.0001$ \\
\hline$<34.9$ & 5770 & $1.08(1.07,1.10)$ & $<0.0001$ & \\
\hline$\geq 34.9$ & 5796 & $1.15(1.13,1.17)$ & $<0.0001$ & \\
\hline Hemoglobin $(\mathrm{g} / \mathrm{dL})$ & & & & $<0.0001$ \\
\hline$<11.6$ & 5699 & $1.09(1.07,1.10)$ & $<0.0001$ & \\
\hline$\geq 11.6$ & 5860 & $1.14(1.13,1.16)$ & $<0.0001$ & \\
\hline Creatinine $(\mathrm{mEq} / \mathrm{L})$ & & & & $<0.0001$ \\
\hline$<1.4$ & 5416 & $1.06(1.04,1.09)$ & $<0.0001$ & \\
\hline$\geq 1.4$ & 6157 & $1.11(1.10,1.13)$ & $<0.0001$ & \\
\hline Glucose (mg/dL) & & & & 0.3993 \\
\hline$<165$ & 5744 & $1.11(1.09,1.13)$ & $<0.0001$ & \\
\hline$\geq 165$ & 5828 & $1.11(1.10,1.13)$ & $<0.0001$ & \\
\hline Chloride $(\mathrm{mmol} / \mathrm{L})$ & & & & 0.0067 \\
\hline$<107$ & 5118 & $1.10(1.09,1.12)$ & $<0.0001$ & \\
\hline$\geq 107$ & 6455 & $1.13(1.11,1.15)$ & $<0.0001$ & \\
\hline $\mathrm{SBP}(\mathrm{mmHg})$ & & & & $<0.0001$ \\
\hline$<114$ & 5763 & $1.13(1.11,1.14)$ & $<0.0001$ & \\
\hline$\geq 114$ & 5777 & $1.08(1.06,1.09)$ & $<0.0001$ & \\
\hline $\mathrm{DBP}(\mathrm{mmHg})$ & & & & 0.0099 \\
\hline$<60$ & 5761 & $1.12(1.11,1.14)$ & $<0.0001$ & \\
\hline$\geq 60$ & 5779 & $1.09(1.08,1.11)$ & $<0.0001$ & \\
\hline $\mathrm{MBP}(\mathrm{mmHg})$ & & & & 0.0004 \\
\hline$<76$ & 5765 & $1.12(1.11,1.14)$ & $<0.0001$ & \\
\hline$\geq 76$ & 5782 & $1.09(1.07,1.11)$ & $<0.0001$ & \\
\hline Heart rate (beats/minute) & & & & 0.5626 \\
\hline$<87$ & 5767 & $1.11(1.09,1.13)$ & $<0.0001$ & \\
\hline$\geq 87$ & 5781 & $1.11(1.10,1.13)$ & $<0.0001$ & \\
\hline
\end{tabular}


TABLE 3: Continued.

\begin{tabular}{|c|c|c|c|c|}
\hline Characteristic & $N$ & HR (95\% CI) & $P$ value & $P$ for interaction \\
\hline Respiratory rate (beats/minute) & & & & 0.4296 \\
\hline$<19$ & 5760 & $1.10(1.08,1.12)$ & $<0.0001$ & \\
\hline$\geq 19$ & 5777 & $1.11(1.09,1.12)$ & $<0.0001$ & \\
\hline Temperature $\left({ }^{\circ} \mathrm{C}\right)$ & & & & 0.0080 \\
\hline$<36.8$ & 5717 & $1.10(1.09,1.11)$ & $<0.0001$ & \\
\hline$\geq 36.8$ & 5725 & $1.12(1.10,1.14)$ & $<0.0001$ & \\
\hline SPO2 (\%) & & & & 0.3747 \\
\hline$<97$ & 5779 & $1.12(1.10,1.13)$ & $<0.0001$ & \\
\hline$\geq 97$ & 5765 & $1.11(1.09,1.12)$ & $<0.0001$ & \\
\hline SOFA score & & & & 0.0513 \\
\hline$<5$ & 4872 & $1.08(1.05,1.11)$ & $<0.0001$ & \\
\hline$\geq 5$ & 6701 & $1.09(1.08,1.10)$ & $<0.0001$ & \\
\hline SAPSII score & & & & 0.0306 \\
\hline$<39$ & 5479 & $1.08(1.05,1.11)$ & $<0.0001$ & \\
\hline$\geq 39$ & 6094 & $1.08(1.07,1.09)$ & $<0.0001$ & \\
\hline RRT & & & & $<0.0001$ \\
\hline No & 10090 & $1.12(1.11,1.14)$ & $<0.0001$ & \\
\hline Yes & 1483 & $1.06(1.04,1.08)$ & $<0.0001$ & \\
\hline
\end{tabular}

CHF: congestive heart failure; CAD: coronary artery disease; AFIB: atrial fibrillation; ARDS: acute respiratory distress syndrome; AKI: acute kidney injury; BUN: blood urea nitrogen; PT: prothrombin time; INR: international normalized ratio; APTT: activated partial thromboplastin time; WBC: white blood cell; SBP: systolic blood pressure; DBP: diastolic blood pressure; MBP: mean blood pressure; SOFA: sequential organ failure assessment; SAPSII: simplified acute physiology score II; RRT: renal replacement therapy.

Similar statistical methods were used to analyze the relationship between lactate, bicarbonate, $\mathrm{pH}$, and urine ketone bodies on the prognosis of AKI patients, as shown in supplementary material. In the fully adjusted model, lactate levels were associated with increased risk 30-day and 90-day all-cause mortality among critically ill patients with AKI, but not with 365-day mortality. As we all know, lactate is closely related to the prognosis of critically ill patients, especially the risk of short-term death $[31,32]$, which can explain the correlation between lactate and short-term prognosis of critically ill patients with AKI. Moreover, bicarbonate, $\mathrm{pH}$, and urine ketone bodies were not independently associated with 30-day, 90-day, and 365-day all-cause mortality. Interestingly, our results further suggested a positive correlation between AG and all-cause mortality in these patients after adjusting for potential confounders such as lactate, $\mathrm{pH}$, and bicarbonate.

There are several limitations in the present study. First, this was a single-center retrospective observational study, and selection bias was inevitable. Second, we measured serum AG in patients only upon admission to the ICU and did not have laboratory follow-up data. There is the possibility of misclassified measured data that may influence the summary results. Third, although we adjusted for confounding factors, our results may have been influenced by other unknown factors. Finally, we could not determine the underlying mechanism between higher AG and poor prognosis; therefore, further study regarding the mechanism is necessary.

\section{Conclusions}

We found that the relationship between AG levels and 30day all-cause mortality was nonlinear, with a U-shaped curve. High-AG levels were associated with increased risk 30-day, 90-day, and 365-day all-cause mortality in critically ill patients with AKI.

\section{Data Availability}

The clinical data used to support the findings of this study were supplied by Monitoring in Intensive Care Database III version 1.4 (MIMIC-III v.1.4). Although the database is publicly and freely available, researchers must complete the National Institutes of Health's web-based course known as Protecting Human Research Participants to apply for permission to access the database.

\section{Disclosure}

The funders of the project were not involved in the study design, collection, data analysis, writing of the report, and publication.

\section{Conflicts of Interest}

The authors declare that they have no competing interests. 


\section{Acknowledgments}

This research was supported by the Scientific Research Foundation of Wenzhou (grant nos. Y20180515 and Y20150038), the Zhejiang Provincial Natural Science Foundation of China (grant nos. LY19H150002 and LY13H150007), and the Clinical Research Foundation of the 2nd Affiliated Hospital of Wenzhou Medical University (SAHoWMU-CR2019-11423).

\section{Supplementary Materials}

Table 1: relationship between bicarbonate and all-cause mortality in different models. Table 2: relationship between $\mathrm{pH}$ and all-cause mortality in different models. Table 3: relationship between lactate and all-cause mortality in different models. Table 4: relationship between urine ketone bodies and all-cause mortality in different models. (Supplementary Materials)

\section{References}

[1] J. S. McDonald, R. J. McDonald, E. E. Williamson, D. F. Kallmes, and K. Kashani, "Post-contrast acute kidney injury in intensive care unit patients: a propensity score-adjusted study," Intensive Care Medicine, vol. 43, no. 6, pp. 774-784, 2017.

[2] A. Odutayo, C. X. Wong, M. Farkouh et al., "AKI and longterm risk for cardiovascular events and mortality," Journal of the American Society of Nephrology, vol. 28, no. 1, pp. 377-387, 2017.

[3] S. Uchino, J. A. Kellum, R. Bellomo et al., "Acute renal failure in critically ill patients: a multinational, multicenter study," JAMA, vol. 294, no. 7, pp. 813-818, 2005.

[4] J. G. M. Hofhuis, H. F. van Stel, A. J. P. Schrijvers, J. H. Rommes, and P. E. Spronk, "The effect of acute kidney injury on long-term health-related quality of life: a prospective follow-up study," Critical Care, vol. 17, no. 1, p. R17, 2013.

[5] L. E. White, H. T. Hassoun, A. Bihorac et al., "Acute kidney injury is surprisingly common and a powerful predictor of mortality in surgical sepsis," The Journal of Trauma and Acute Care Surgery, vol. 75, no. 3, pp. 432-438, 2013.

[6] Y. Hu, H. Liu, S. Fu, J. Wan, and X. Li, "Red blood cell distribution width is an independent predictor of AKI and mortality in patients in the coronary care unit," Kidney \& Blood Pressure Research, vol. 42, no. 6, pp. 1193-1204, 2017.

[7] B. Wu, J. Chen, and Y. Yang, "Biomarkers of acute kidney injury after cardiac surgery: a narrative review," BioMed Research International, vol. 2019, Article ID 7298635, 11 pages, 2019.

[8] J. A. Kraut and N. E. Madias, "Serum anion gap: its uses and limitations in clinical medicine," Clinical Journal of the American Society of Nephrology, vol. 2, no. 1, pp. 162-174, 2007.

[9] M. M. Salem and S. K. Mujais, "Gaps in the anion gap," Archives of Internal Medicine, vol. 152, no. 8, pp. 1625-1629, 1992.

[10] W. R. Farwell and E. N. Taylor, "Serum bicarbonate, anion gap and insulin resistance in the National Health and Nutrition Examination Survey," Diabetic Medicine, vol. 25, no. 7, pp. 798-804, 2008.
[11] M. K. Abramowitz, T. H. Hostetter, and M. L. Melamed, "Lower serum bicarbonate and a higher anion gap are associated with lower cardiorespiratory fitness in young adults," Kidney International, vol. 81, no. 10, pp. 1033-1042, 2012.

[12] M. Park, S. J. Jung, S. Yoon, J. M. Yun, and H. J. Yoon, “Association between the markers of metabolic acid load and higher all-cause and cardiovascular mortality in a general population with preserved renal function," Hypertension Research, vol. 38, no. 6, pp. 433-438, 2015.

[13] N. M. Mohr, J. P. Vakkalanka, B. A. Faine et al., "Serum anion gap predicts lactate poorly, but may be used to identify sepsis patients at risk for death: a cohort study," Journal of Critical Care, vol. 44, pp. 223-228, 2018.

[14] S. W. Yang, Y. J. Zhou, Y. X. Zhao et al., "The serum anion gap is associated with disease severity and all-cause mortality in coronary artery disease," Journal of Geriatric Cardiology, vol. 14, no. 6, pp. 392-400, 2017.

[15] Q. Chen, Q. Chen, L. Li et al., "Serum anion gap on admission predicts intensive care unit mortality in patients with aortic aneurysm," Experimental and Therapeutic Medicine, vol. 16, no. 3, pp. 1766-1777, 2018.

[16] M. K. Abramowitz, T. H. Hostetter, and M. L. Melamed, "The serum anion gap is altered in early kidney disease and associates with mortality," Kidney International, vol. 82, no. 6, pp. 701-709, 2012.

[17] B. Wang, D. Li, Y. Gong, B. Ying, and B. Cheng, "Association of serum total and ionized calcium with all-cause mortality incritically ill patients with acute kidney injury," Clinica Chimica Acta, vol. 494, pp. 94-99, 2019.

[18] B. Wang, Y. Gong, B. Ying, and B. Cheng, "Relation between red cell distribution width and mortality in critically ill patients with acute respiratory distress syndrome," BioMed Research International, vol. 2019, Article ID 1942078, 8 pages, 2019.

[19] B. Wang, H. Lu, Y. Gong, B. Ying, and B. Cheng, "The association between red blood cell distribution width and mortality in critically ill patients with acute kidney injury," BioMed Research International, vol. 2018, Article ID 9658216, 7 pages, 2018.

[20] A. E. W. Johnson, T. J. Pollard, L. Shen et al., "MIMIC-III, a freely accessible critical care database," Scientific Data, vol. 3, no. 1, article 160035, 2016.

[21] J. Allard, S. Cotin, F. Faure et al., "SOFA-an open source framework for medical simulation," Studies in Health Technology and Informatics, vol. 125, pp. 13-18, 2007.

[22] J. R. Le Gall, S. Lemeshow, and F. Saulnier, "A new simplified acute physiology score (SAPS II) based on a European/North American multicenter study," JAMA, vol. 270, no. 24, pp. 2957-2963, 1993.

[23] T. Agoritsas, A. Merglen, N. D. Shah, M. O'Donnell, and G. H. Guyatt, "Adjusted analyses in studies addressing therapy and harm: users' guides to the medical literature," JAMA, vol. 317, no. 7, pp. 748-759, 2017.

[24] T. Banerjee, D. C. Crews, D. E. Wesson et al., "Elevated serum anion gap in adults with moderate chronic kidney disease increases risk for progression to end-stage renal disease," American Journal of Physiology-Renal Physiology, vol. 316, no. 6, pp. F1244-f1253, 2019.

[25] M. Al-Jaghbeer and J. A. Kellum, “Acid-base disturbances in intensive care patients: etiology, pathophysiology and treatment," Nephrology, Dialysis, Transplantation, vol. 30, no. 7, pp. 1104-1111, 2015. 
[26] P. A. Gabow, W. D. Kaehny, P. V. Fennessey, S. I. Goodman, P. A. Gross, and R. W. Schrier, "Diagnostic importance of an increased serum anion gap," The New England Journal of Medicine, vol. 303, no. 15, pp. 854-858, 1980.

[27] L. S. Chawla, R. Bellomo, A. Bihorac et al., "Acute kidney disease and renal recovery: consensus report of the acute disease quality initiative (ADQI) 16 workgroup," Nature Reviews Nephrology, vol. 13, no. 4, pp. 241-257, 2017.

[28] D. Q. Sun, L. Zhang, C. F. Zheng et al., "Metabolic acidosis in critically ill cirrhotic patients with acute kidney injury," Journal of Clinical and Translational Hepatology, vol. 7, no. 2, pp. 112-121, 2019.

[29] B. Levy, S. Gibot, P. Franck, A. Cravoisy, and P. E. Bollaert, "Relation between muscle $\mathrm{Na}^{+} \mathrm{K}^{+}$ATPase activity and raised lactate concentrations in septic shock: a prospective study," The Lancet, vol. 365, no. 9462, pp. 871-875, 2005.

[30] I. Kurtz, T. Maher, H. N. Hulter, M. Schambelan, and A. Sebastian, "Effect of diet on plasma acid-base composition in normal humans," Kidney International, vol. 24, no. 5, pp. 670-680, 1983.

[31] J.-L. Vincent, A. Quintairos e Silva, L. Couto, and F. S. Taccone, "The value of blood lactate kinetics in critically ill patients: a systematic review," Critical Care, vol. 20, no. 1, p. 257, 2016.

[32] J. Seheult, G. Fitzpatrick, and G. Boran, "Lactic acidosis: an update," Clinical Chemistry and Laboratory Medicine, vol. 55, no. 3, pp. 322-333, 2017. 


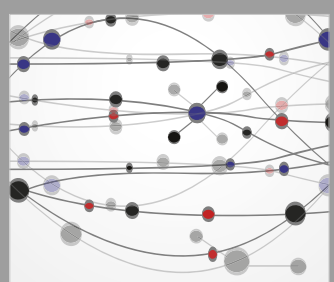

The Scientific World Journal
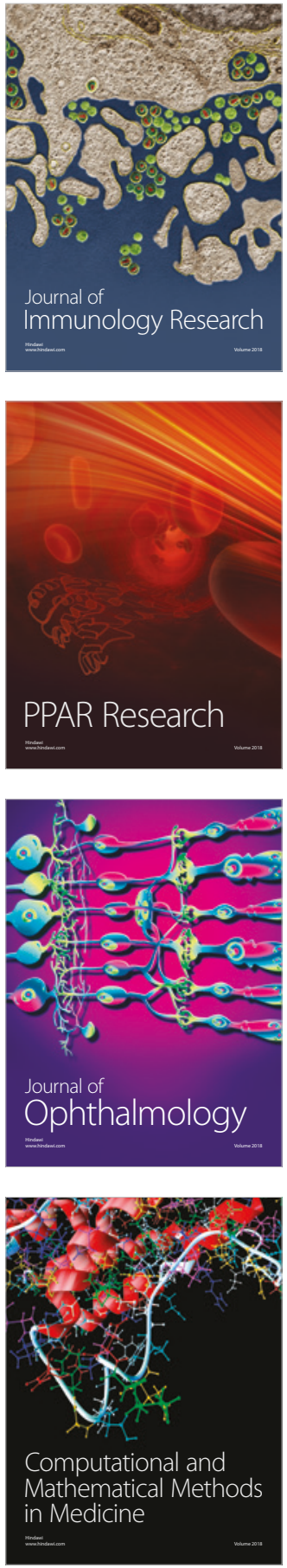

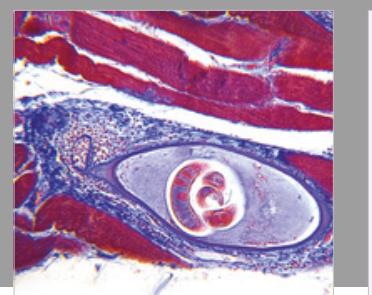

Gastroenterology Research and Practice

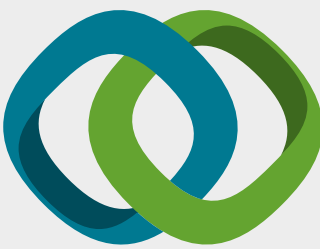

\section{Hindawi}

Submit your manuscripts at

www.hindawi.com
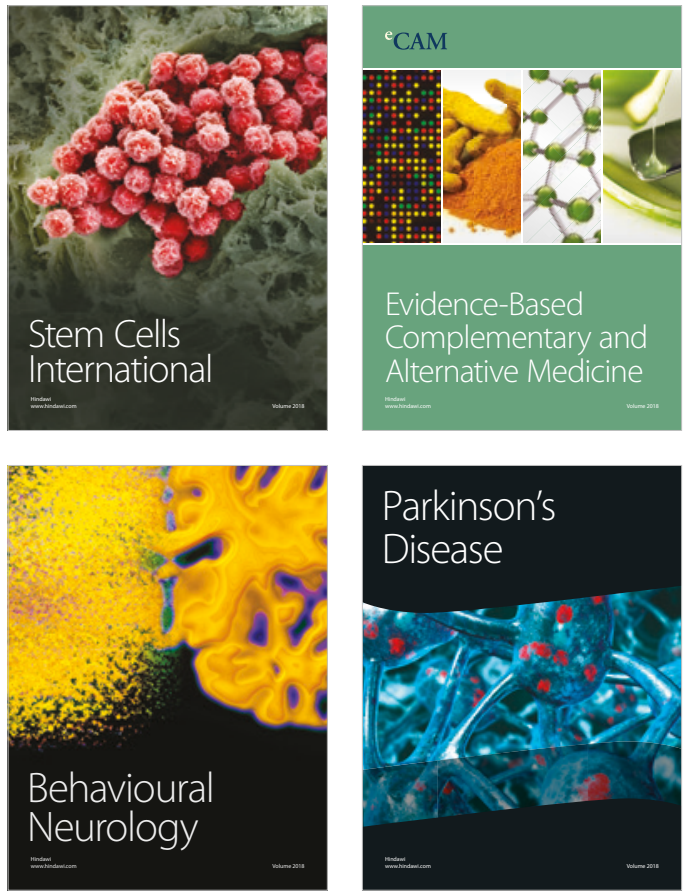

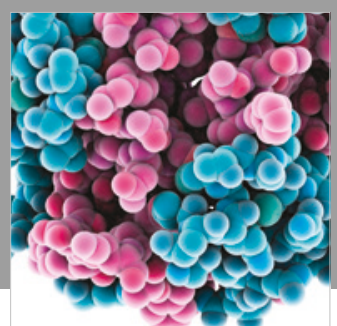

ournal of

Diabetes Research

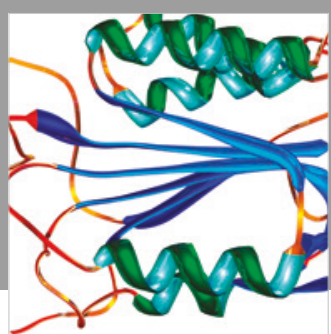

Disease Markers
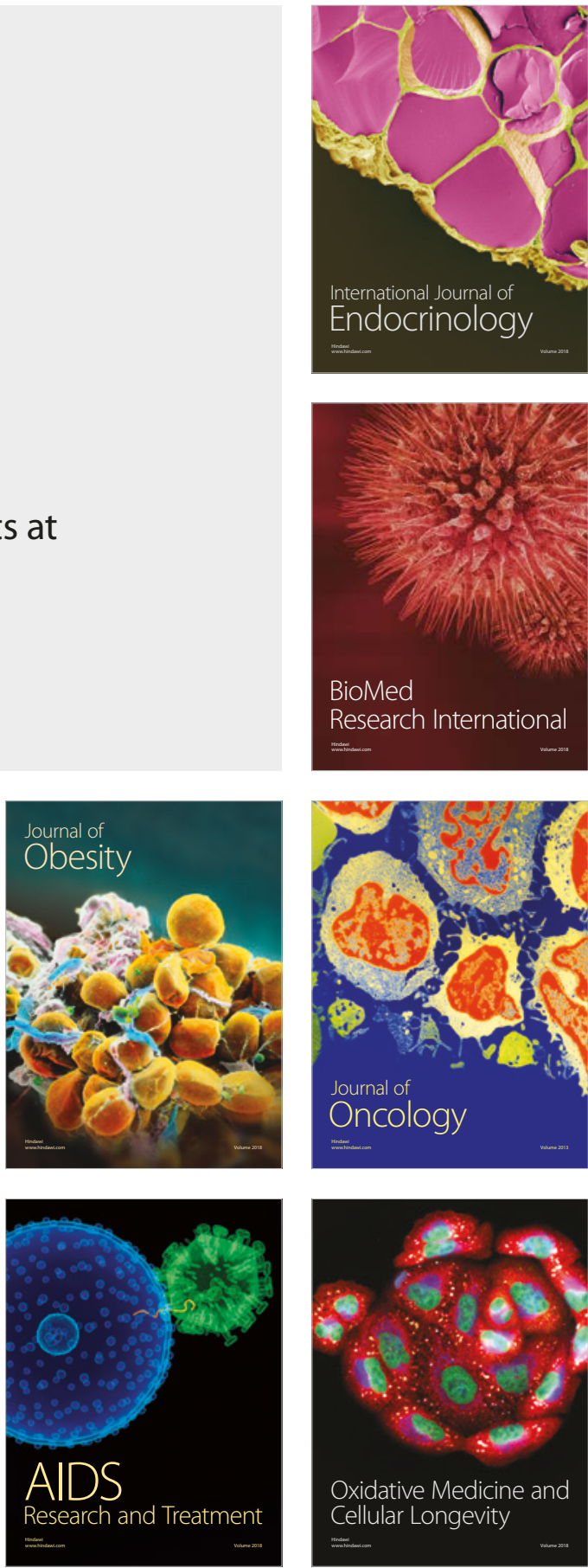